\title{
Pain-Induced Analgesia Mediated by Mesolimbic Reward Circuits
}

\author{
Robert W. Gear, ${ }^{1,2}$ K. O. Aley, ${ }^{2}$ and Jon D. Levine ${ }^{2-6}$ \\ ${ }^{1}$ Center for Orofacial Pain, Departments of ${ }^{2}$ Oral and Maxillofacial Surgery, ${ }^{3}$ Anatomy, and ${ }^{4}$ Medicine, \\ ${ }^{5}$ Division of Neuroscience, and ${ }^{6}$ National Institutes of Health Pain Center (UCSF), University of California, \\ San Francisco, California 94143
}

\begin{abstract}
We tested the hypothesis that noxious stimuli induce pain modulation by activation of supraspinal structures. We found that intense noxious stimuli (i.e., subdermal injection of capsaicin or paw immersion in hot water) induced profound attenuation of the jaw-opening reflex in the anesthetized rat; forepaw subdermal capsaicin also elevated the mechanical hindpawwithdrawal threshold in the awake rat. These antinociceptive effects were blocked by previous injection of either a dopamine antagonist (flupentixol) or an opioid antagonist (naloxone) into the nucleus accumbens. Additional experiments in anesthetized animals showed that the antinociceptive effect of noxious stimulation by either capsaicin $(\geq 100 \mu \mathrm{g})$ or hindpaw immersion in hot water $\left(\geq 45^{\circ} \mathrm{C}\right.$ for $4 \mathrm{~min}$ ) correlated with the intensity of the stimulus. The maximal antinociceptive effect of capsaicin
\end{abstract}

was similar in magnitude to that of a high dose of morphine (10 $\mathrm{mg} / \mathrm{kg}$ ) injected subcutaneously. Injection of the $\mathrm{GABA}_{\mathrm{A}^{-}}$ receptor agonist muscimol, but not naloxone, into the rostroventral medulla, a major component of descending pain modulation systems, blocked capsaicin-induced antinociception. Although it is widely thought that painful stimuli may induce analgesia by activating forebrain structures, this is the first demonstration that such a mechanism exists. Furthermore, this mechanism can be engaged by naturalistic stimuli in awake animals. These observations imply that painful stimuli might under certain conditions be rewarding.

Key words: antinociception; capsaicin; nociception; noxious stimuli; nucleus accumbens; rostroventral medulla; opioids; dopamine; thermal stimulation; GABA; rats
The nucleus accumbens, which is known to be an important component of the mesolimbic dopaminergic reward system and has been implicated in substance abuse, also plays a role in pain modulation. However, although microinjection of opioid directly into nucleus accumbens has been shown to induce antinociception (Dill and Costa, 1977; Yu and Han, 1989; Tseng and Wang, 1992) and microinjection of the opioid antagonist naloxone into nucleus accumbens has been shown to attenuate the antinociceptive effect of systemically administered morphine (Dill and Costa, 1977; Daghero et al., 1987), the physiological mechanisms underlying the role of nucleus accumbens in nociception are poorly understood. We recently demonstrated that nucleus accumbens opioidergic mechanisms participate in a novel ascending nociceptive control circuit (Gear and Levine, 1995); that is, intraaccumbens microinjection of naloxone blocks the antinociceptive effect of pharmacological interventions made at the level of the spinal cord. Because this circuit could be activated by spinal interventions, we hypothesized that peripheral sensory input, such as noxious stimulation, might be a mechanism by which the ascending nociceptive control is physiologically activated. Therefore, in the present study, we tested the hypothesis that activation of nociceptors in the paw would produce heterosegmental antinociception via the circuitry in nucleus accumbens mediating the

Received March 25, 1999; revised June 2, 1999; accepted June 4, 1999.

This work was supported by the State of California Tobacco-Related Diseases Research program. We are grateful to Drs. Michael Gold, David Reichling, Philip Heller, Kimberly Tanner, and Holly Strausbaugh for many helpful discussions during the course of this work. We also thank Justine Barletta and Alexander Riedel for excellent technical assistance.

Correspondence should be addressed to Dr. Jon D. Levine, National Institutes of Health Pain Center (UCSF), C-522 (Box 0440), University of California, San Francisco, CA 94143-0440.

Copyright (C) 1999 Society for Neuroscience $\quad 0270-6474 / 99 / 197175-07 \$ 05.00 / 0$ ascending nociceptive control. Because nucleus accumbens dopaminergic mechanisms may play a role in pain modulation (Altier and Stewart, 1993), we also tested their involvement in noxious stimulus-induced antinociception.

The descending pathway for antinociception mediated by the nucleus accumbens might include the well known periaqueductal gray (PAG)-rostroventral medulla (RVM)-spinal cord pain modulation system. Pain modulation circuits in the PAG project to the RVM, which contains both opioidergic and GABAergic circuitry also important in pain modulation (Fields et al., 1991). The RVM, in turn, sends projections to the spinal cord dorsal horn (Fields et al., 1977; Fields and Basbaum, 1978; Cho and Basbaum, 1991) that attenuate nociceptive signals originating in the periphery. The widely held expectation that noxious stimuli can induce analgesia by activating forebrain structures (Basbaum and Fields, 1978) has eluded demonstration, even after more than two decades of research. Therefore, we also tested the hypothesis that the RVM contributes to noxious stimulus-induced antinociception.

\section{MATERIALS AND METHODS}

Experiments were performed on 280-380 gm male Sprague Dawley rats (Bantin and Kingman, Fremont, CA). These animals were maintained in the University of California, San Francisco (UCSF), animal care facility in accordance with applicable university policies. Experimental protocols were approved by the UCSF Committee on Animal Research. Antinociception in awake animals was measured as elevation of threshold for withdrawal of the hindpaw from an increasing mechanical stimulus. Antinociception in anesthetized animals was measured as attenuation of the nociceptive jaw-opening reflex (Mason et al., 1985; Gear and Levine, 1995) (Fig. 1).

Anesthesia. In some experiments, animals were anesthetized by intraperitoneal injection of $0.9 \mathrm{gm} / \mathrm{kg}$ urethane and $45 \mathrm{mg} / \mathrm{kg} \alpha$-chloralose (both from Sigma, St. Louis, MO). Urethane- $\alpha$-chloralose was chosen for anesthesia because this anesthetic protocol provides a stable jaw- 


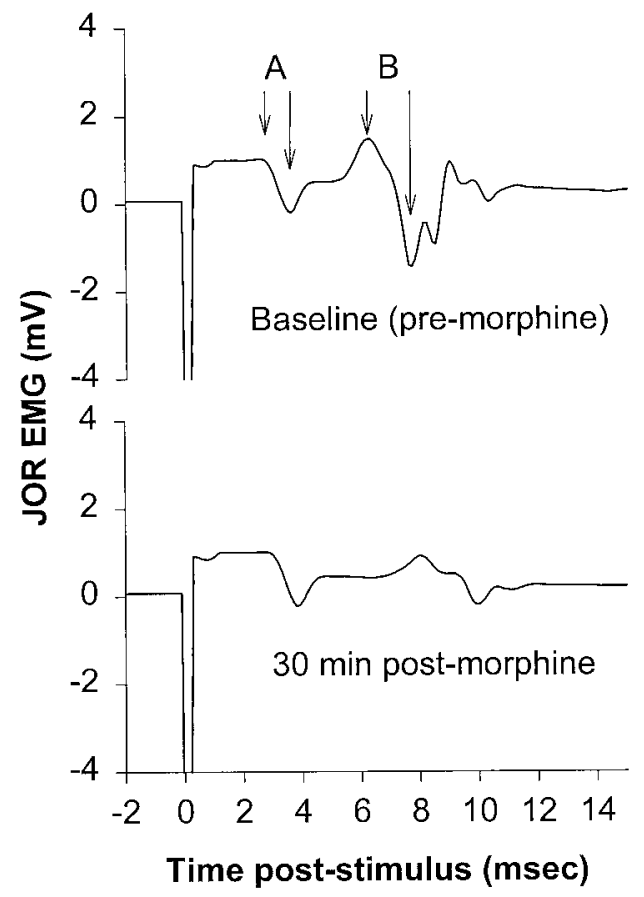

Figure 1. Example of jaw-opening reflex EMG tracing before and after administration of morphine. The top trace demonstrates two components, $A$ and $B$, which show EMG amplitudes of 1.0 and $2.9 \mathrm{mV}$, respectively. Based on the short latency, component $A$ results from activation of fast-conducting (presumably non-nociceptive) afferents; the latency of component $B$ is compatible with the conduction velocity of $\mathrm{A} \delta$ nociceptors (calculated conduction velocity of $\sim 16-20 \mathrm{~m} / \mathrm{sec}$ ). The bottom trace, recorded from the same animal 30 min after systemic administration of morphine $(10 \mathrm{mg} / \mathrm{kg}$, i.p.), shows attenuation of component $B$ but no change in component $A$, compatible with the findings of others that opioids do not attenuate non-nociceptive responses. The data in this study were always taken from that part of the EMG signal exhibiting a latency similar to that of component $B$.

opening reflex electromyographic (EMG) signal over the time period required to complete the experiments (Gear and Levine, 1995).

Electrode implantation. To evoke the jaw-opening reflex, a bipolar stimulating electrode, fabricated from two insulated copper wires (36 AWG), each with $0.2 \mathrm{~mm}$ of insulation removed from the tip, one tip extending $2 \mathrm{~mm}$ beyond the other, was inserted into the pulp of a mandibular incisor to a depth of $22 \mathrm{~mm}$ from the incisal edge of the tooth to the tip of the longest wire and cemented into place with dental composite resin (Citrix; Golden Gate Dental Supply Inc., South San Francisco, CA). A bipolar recording electrode, consisting of two wires of the same material as the stimulating electrode with $4 \mathrm{~mm}$ of insulation removed, was inserted into the digastric muscle ipsilateral to the implanted tooth to a depth sufficient to completely submerge the uninsulated end of the wire.

Jaw-opening reflex. At the beginning of each experiment, stimulation current was set at three times the threshold current. Changes in nociception were measured as changes in jaw-opening reflex EMG signal amplitude (Gear and Levine, 1995) (Fig. 1). Each data point consisted of the average peak-to-peak amplitude of 12 consecutive jaw-opening reflex EMG signals evoked by stimulating the tooth pulp with $0.2 \mathrm{msec}$ square wave pulses at a frequency of $0.33 \mathrm{~Hz}$. Baseline amplitude was defined as the average of the last three data points, recorded at 5 min intervals, before an experimental intervention. Effects of experimental interventions are expressed as the mean \pm SEM percentage change from the baseline for each experimental group. Repeated measures ANOVA with the Scheffé post hoc test or the Student-Newman-Keuls test were used to compare groups for significant differences $(p \leq 0.05)$.

Paw-withdrawal test. Awake rats were gently restrained (Taiwo et al., 1989), allowing mechanical stimulation to the hindpaws (Randall-Selitto Analgesimeter; Ugo Basile). Thresholds for withdrawal were measured for both hindpaws immediately before the administration of capsaicin and again 20, 25, and 30 min afterward. Baseline scores were calculated as the average of the precapsaicin thresholds for the two hindpaws. Postcapsaicin thresholds were also averaged to obtain a single score for each animal. Effects of experimental interventions are expressed as the mean \pm SEM percentage change (in grams) from the baseline for each experimental group.

Cannula placement. For nucleus accumbens, RVM, and PAG injections, 25 gauge (23 gauge in awake animals) stainless steel guide cannulas were stereotactically positioned and cemented with orthodontic resin (L.D. Caulk Co., Milford, DE) to allow injections via insertion of a 33 gauge (30 gauge in awake animals) stainless steel injection cannula, which extended beyond the guide cannulas $4 \mathrm{~mm}$, connected to a $2 \mu \mathrm{l}$ syringe (Hamilton, Reno, NV). Supraspinal injection volumes were 0.3 $\mu \mathrm{l}$ in all experiments. These injections were performed over a period of $90 \mathrm{sec}$, and the injection cannulas were left in place an additional $30 \mathrm{sec}$ after injection. The stereotaxic instrument was set to the following coordinates for onsite injections: nucleus accumbens (from bregma), 1.2 $\mathrm{mm}$ rostral, $7.5 \mathrm{~mm}$ ventral, and $\pm 1.8 \mathrm{~mm}$ lateral; $\mathrm{RVM}$ (from intra-aural line), $2.3 \mathrm{~mm}$ caudal, $-0.6 \mathrm{~mm}$ ventral, and $\pm 1 \mathrm{~mm}$ lateral from the midline for bilateral injections or midline for single injections. Injection sites were verified by histological examination $(50 \mu \mathrm{m}$ sections stained with cresyl violet acetate) and were plotted on coronal sections adapted from the atlas of Paxinos and Watson (1986).

Drugs. Capsaicin (Sigma, St. Louis, MO) was dissolved in Tween 80 $(50 \%)$ and ethanol $(50 \%)$ to an initial concentration of $50 \mu \mathrm{g} / \mu \mathrm{l}$ and was diluted with PBS as necessary to obtain the desired dose for each experiment. Subdermal capsaicin injection volume was $50 \mu \mathrm{l}$ in all experiments. Naloxone methiodide, a quaternary form of naloxone, flupentixol, a nonselective dopamine receptor antagonist, and muscimol, a GABA $_{A}$-receptor agonist (Research Biochemicals, Natick, MA) were dissolved in PBS. Bupivacaine $(0.5 \%$; Abbott Laboratories, North Chicago, IL) was administered in two adjacent $50 \mu$ injections to block primary afferent activity in the area of capsaicin injection. Morphine (Elkins-Sinn Inc., Cherry Hill, NJ), diluted as necessary with $0.9 \%$ saline, was injected subcutaneously in the midback; injection volumes were 1 $\mathrm{ml} / \mathrm{kg}$ body weight. [D-Ala ${ }^{2}, N$-Me-Phe ${ }^{4}, \mathrm{Gly}^{5}$-ol]-enkephalin (DAMGO) (Sigma) was dissolved in $0.9 \%$ saline.

Statistics. Repeated measures ANOVA with the Scheffé post hoc test or the Student-Newman-Keuls test was used as appropriate to compare groups for significant differences $(p \leq 0.05)$.

\section{RESULTS}

Heterosegmental noxious stimulus-induced antinociception was demonstrated by the ability of capsaicin, injected into the plantar surface of a hindpaw, to dose-dependently attenuate the jawopening reflex (Fig. 2A,B). The maximal antinociceptive effect of capsaicin was produced by doses $\geq 250 \mu \mathrm{g}$, which attenuated the jaw-opening reflex $>60 \%$ (Fig. $2 B$ ). To compare the potency of capsaicin-induced antinociception with that of a standard opioid analgesic, attenuation of the jaw-opening reflex by systemically administered morphine was evaluated in separate groups of animals (Fig. 2A). The maximal antinociceptive effect of capsaicin was similar to that of a high dose of morphine $(10 \mathrm{mg} / \mathrm{kg})$.

The antinociceptive effect of capsaicin was blocked by injection of the local anesthetic bupivacaine into the hindpaw $15 \mathrm{~min}$ before the administration of capsaicin into the same location (Fig. $2 C$ ), indicating lack of a systemic effect by capsaicin. The same dose of bupivacaine injected into the contralateral paw had no effect on antinociception (data not shown). Administration of bupivacaine $10 \mathrm{~min}$ subsequent to capsaicin did not reverse antinociception (Fig. 2C), indicating that the prolonged antinociceptive effect does not depend on ongoing afferent input. Bilateral hindpaw immersion in a hot water bath for 4 min at various temperatures $\left(40,45\right.$, and $\left.50^{\circ} \mathrm{C}\right)$ revealed a temperaturedependent attenuation of the jaw-opening reflex (Fig. $2 D$ ), indicating that heterosegmental antinociception can be induced by thermal, as well as chemical, noxious stimuli. 
A
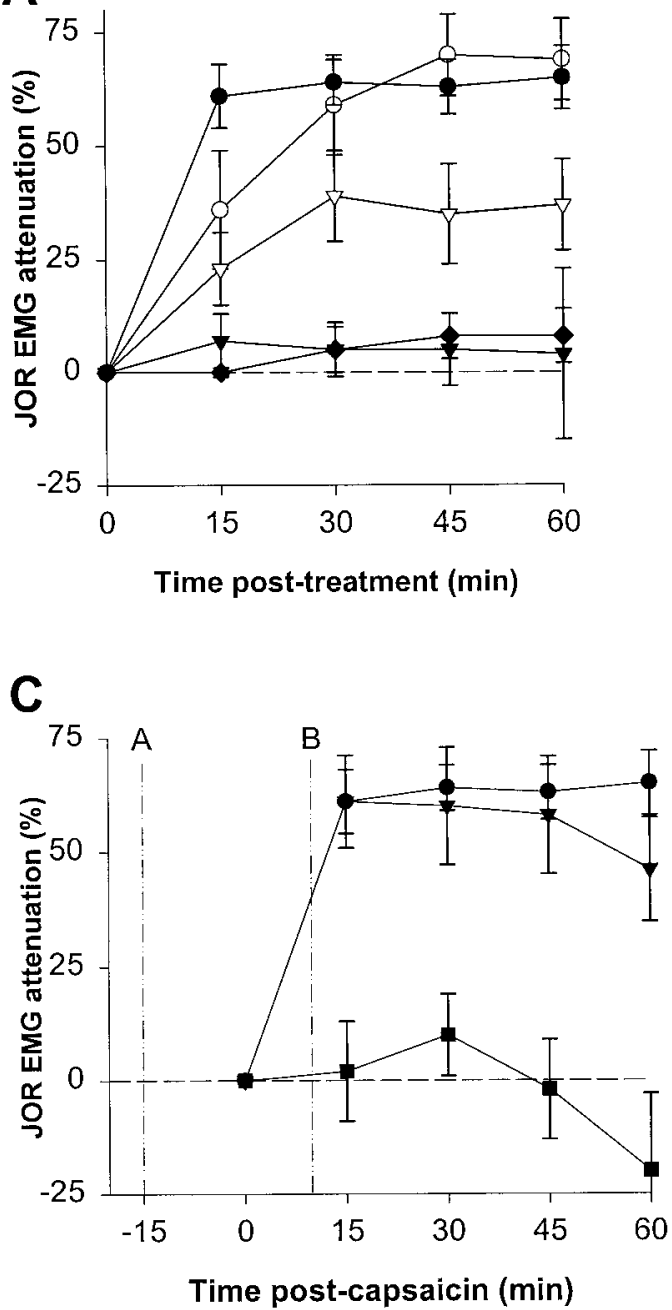

B

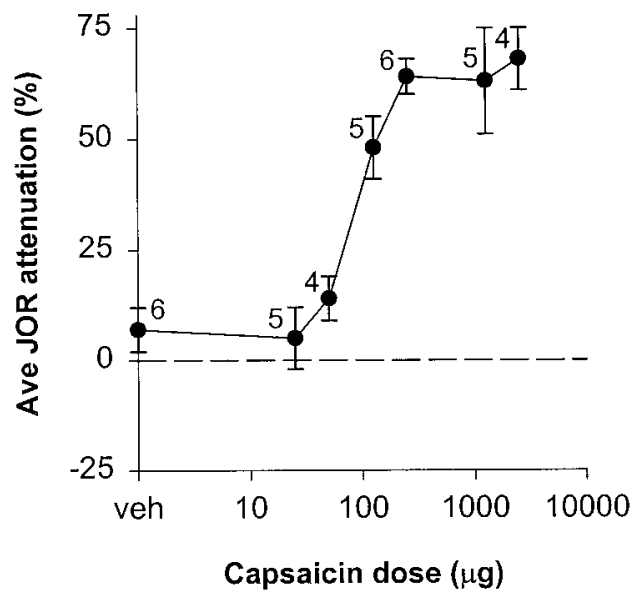

D

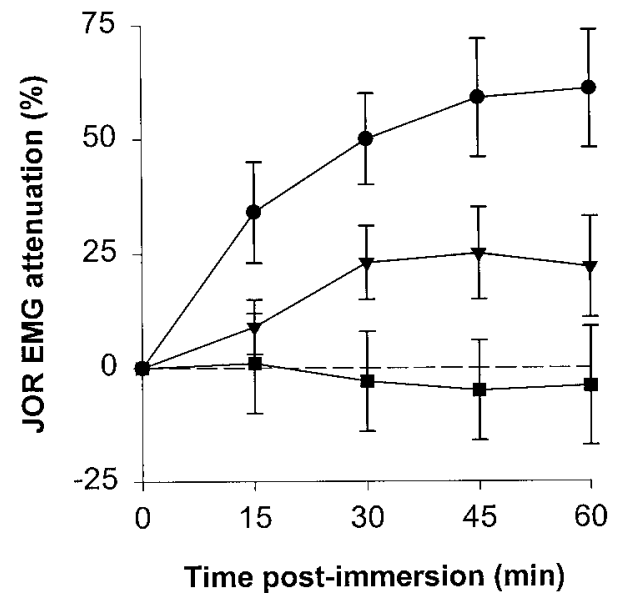

Figure 2. The antinociceptive effect of noxious stimulation. A, Effect of capsaicin or morphine on the jaw-opening reflex EMG plotted over time. Note that the $25 \mu \mathrm{g}$ dose $(\boldsymbol{\nabla})(n=6)$ did not significantly affect the jaw-opening reflex, whereas the $250 \mu \mathrm{g}$ dose of capsaicin $(\boldsymbol{\bullet})(n=6)$ attenuated the jaw-opening reflex $>60 \%$. This high dose of capsaicin induced significantly greater antinociception than the $5 \mathrm{mg} / \mathrm{kg} \mathrm{dose}$ of morphine $(\nabla)(n=7)$ but was not significantly different from the maximal antinociceptive effect of $10 \mathrm{mg} / \mathrm{kg}$ morphine $(\bigcirc)(n=6)$; the slower onset of morphine antinociception was presumably caused by the subcutaneous route of administration. The stability of the jaw-opening reflex over time is indicated by lack of significant change from baseline of the group receiving capsaicin vehicle $(\bullet)(n=6)$. $B$, Average attenuation of the jaw-opening reflex over $1 \mathrm{hr}$ induced by various doses of capsaicin (including the two doses plotted in $A$ ). Number of animals of each group is indicated next to each data point. $C$, The effect of the local anesthetic bupivacaine on capsaicin-induced antinociception. Bupivacaine was administered either 15 min before $(A, \boldsymbol{\square})(n=6)$ or 10 min after $(B, \boldsymbol{\nabla})$ $(n=6)$ capsaicin $(250 \mu \mathrm{g})$ to the same site. For comparison, the effect of the same dose of capsaicin administered as a single agent is replotted from $A(\bullet) . D$, The effect on the jaw-opening reflex of immersion of both hindpaws into water at various temperatures for 4 min. The last baseline jaw-opening reflex was recorded immediately before immersion; the first postimmersion recording was 15 min after removal of the paws from the bath. $40^{\circ} \mathrm{C}, \boldsymbol{\square}(n=$ $4) ; 45^{\circ} \mathrm{C}, \boldsymbol{\nabla}(n=6) ; 50^{\circ} \mathrm{C},(n=5)$. In this and subsequent figures, the data are plotted as mean $\pm \mathrm{SEM}$.

Administration of either naloxone methiodide $(1 \mu \mathrm{g}$ bilaterally), or flupentixol ( $3 \mu \mathrm{g}$ bilaterally) into the nucleus accumbens 15 min before intraplantar capsaicin injection blocked antinociception (Fig. $3 A$; see Fig. $5 A$ for injection sites). Antinociception induced by a noxious thermal stimulus was also blocked by either naloxone methiodide or flupentixol (same doses) administered into the nucleus accumbens $15 \mathrm{~min}$ before stimulation (Fig. 3B). Injections of the same doses of either naloxone methiodide or flupentixol near by but not in nucleus accumbens did not block capsaicin-induced antinociception (Fig. 3C). Also, when administered in the absence of noxious stimulation, neither nucleus accumbens injection of naloxone methiodide nor flupentixol affected the jaw-opening reflex (Fig. $3 C$ ).
To confirm these results in awake animals using a different nociceptive assay, the effect on the paw-withdrawal reflex of capsaicin $(125 \mu \mathrm{g})$ injected into a forepaw was determined. Thirty minutes after capsaicin injection, the paw-withdrawal threshold was elevated $64 \%$, and this effect was blocked by previous administration of either naloxone or flupentixol into the nucleus accumbens (Fig. 3D; see Fig. $5 A$ for injection sites).

The involvement of a descending pain modulation system in noxious stimulation-induced antinociception was assessed by injecting either the $\mathrm{GABA}_{\mathrm{A}}$-receptor agonist muscimol $(10 \mathrm{ng}$, single midline injection) or naloxone methiodide (1 $\mu \mathrm{g}$ bilaterally) into the RVM 10 or 15 min, respectively, before the administration of capsaicin to the hindpaw. Muscimol, but not naloxone 

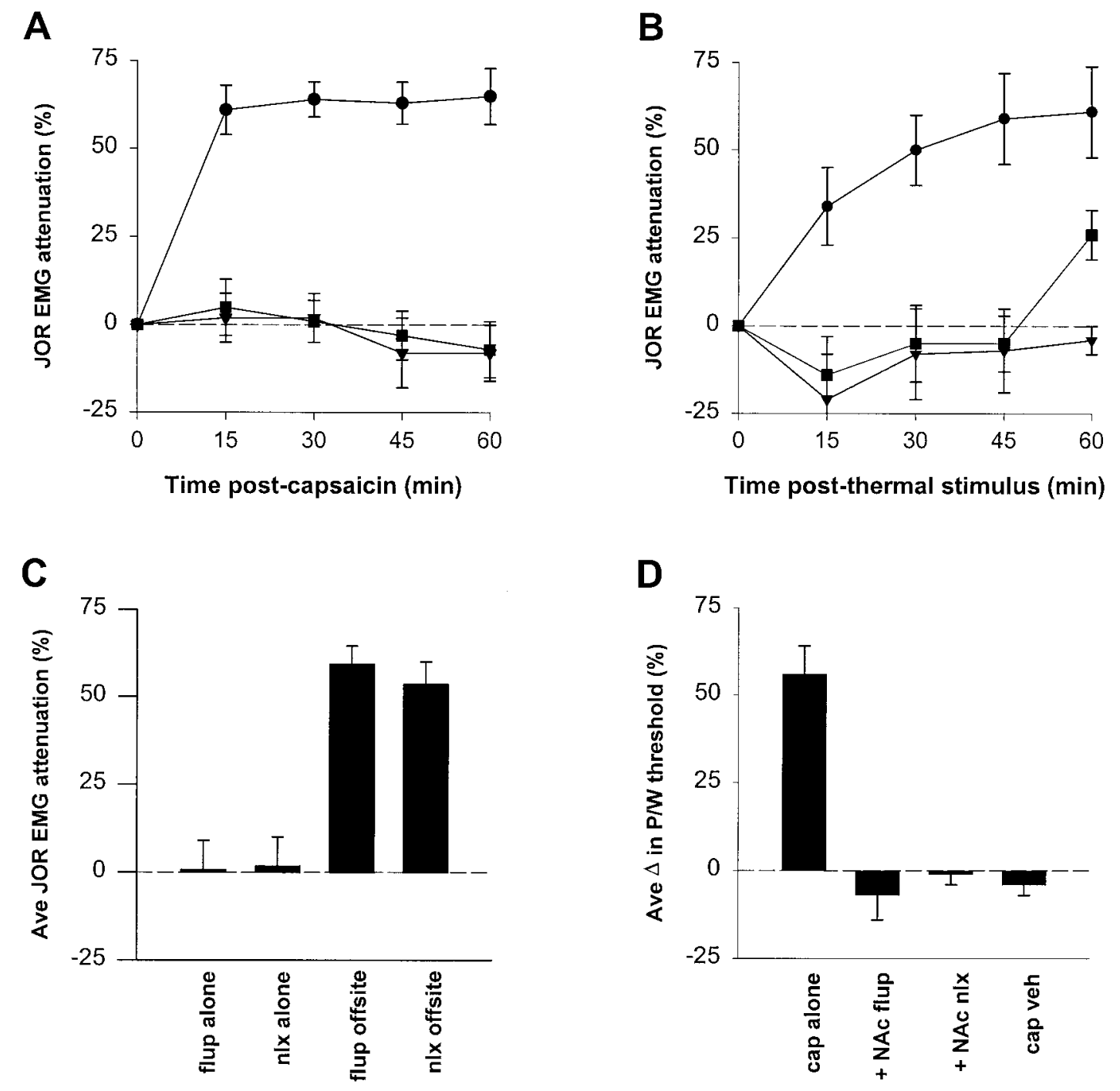

Figure 3. The effect a dopamine or opioid antagonist on noxious stimulus-induced antinociception. All pretreatments were given 15 min before the onset of noxious stimulation. $A$, Naloxone methiodide (opioid antagonist, $\boldsymbol{\nabla})(n=5)$ or flupentixol (dopamine antagonist, $\mathbf{\square})(n=6)$ was injected into nucleus accumbens before injection of capsaicin $(250 \mu \mathrm{g})$ in the hindpaw. The effect of capsaicin injection without pretreatment (same dose, $)$ is replotted from Figure $2 A$. $B$, Naloxone methiodide $(\boldsymbol{\nabla})(n=6)$ or flupentixol $(\boldsymbol{\square})(n=5)$ was injected into nucleus accumbens before thermal stimulation (both hindpaws immersed in water, $50^{\circ} \mathrm{C}, 4 \mathrm{~min}$ ). The effect of thermal stimulation without pretreatment (same stimulus intensity, 0 ) is replotted from Figure 2C. $C$, The effect of either flupentixol ( flup alone) $(n=6)$ or naloxone methiodide (nlx alone) $(n=6)$ injected into nucleus accumbens as a single agent; the effect on capsaicin-induced antinociception of previous injection of either flupentixol ( flup offsite) $(n=6)$ or naloxone methiodide (nlx offsite) $(n=6)$ administered to sites adjacent to nucleus accumbens. $D$, The effect of capsaicin administered to the forepaw on hindpaw-withdrawal threshold in awake animals when administered either alone (cap alone) $(n=6)$ or in the presence of previous administration of flupentixol $(+N A c$ flup $)(n=5)$ or naloxone methiodide $(+N A c n l x)(n=5)$. Capsaicin vehicle with no supraspinal treatment was also administered $(c a p$ veh $)(n=4)$. Antinociception is indicated by increased threshold for withdrawal.

methiodide, blocked attenuation of the jaw-opening reflex (Figs. 4, $5 B$, injection sites), indicating that RVM GABAergic, but not opioidergic, circuits mediate noxious stimulus-induced antinociception. Muscimol injected into the RVM did not itself significantly affect the jaw-opening reflex (Figs. 4, 5B, injection sites). However, compatible with the findings of others (Kiefel et al., 1993; Roychowdhury and Fields, 1996), a similar injection of naloxone into the RVM did block antinociception by intra-PAG injection of the $\mu$-opioid DAMGO (60 ng) (Figs. 4, 5B, injection sites). These results indicate that a descending pain modulation system at the level of the RVM mediates noxious stimulusinduced antinociception, but that circuits descending from the
PAG to the RVM that are mediated by endogenous opioids in the RVM do not appear to be involved.

\section{DISCUSSION}

The results of this study demonstrate that activation of primary afferent neurons by noxious stimulation in the form of either capsaicin administration or paw immersion in hot water induces heterosegmental antinociception in both awake and anesthetized animals. The potency of this noxious stimulus-induced antinociception is indicated by its ability to produce attenuation of the jaw-opening reflex to a degree similar to that of high-dose (10 $\mathrm{mg} / \mathrm{kg}$ ) systemically administered morphine. 


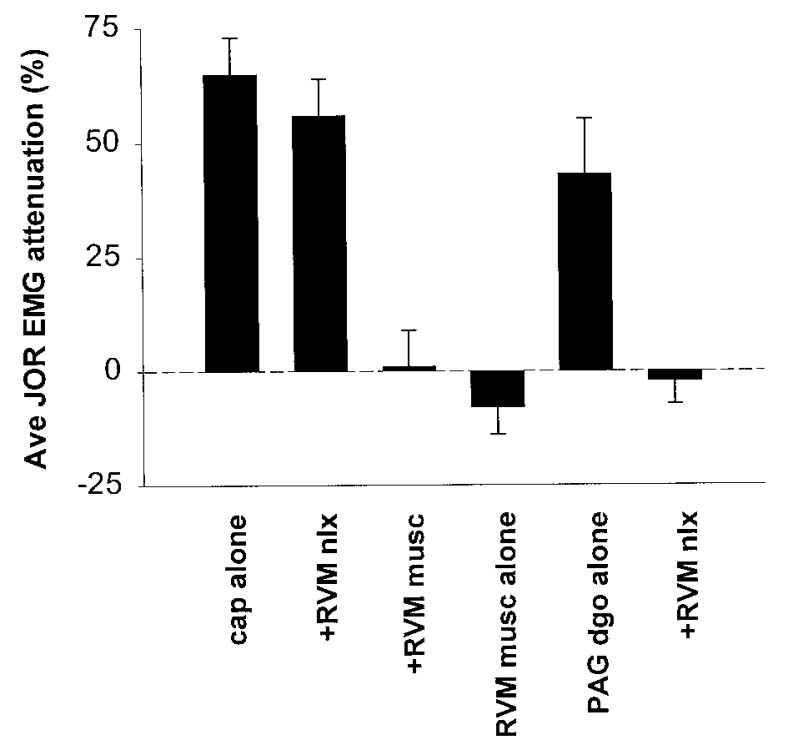

Figure 4. The effect of either naloxone methiodide or muscimol administered to the RVM on attenuation of the jaw-opening reflex induced by either intraplantar capsaicin or intra-PAG DAMGO. Each bar represents the average of four recordings taken at $15 \mathrm{~min}$ intervals for $1 \mathrm{hr}$. Left to right, Intraplantar capsaicin administered as a single agent (cap alone) (data from Fig. 3A), intra-RVM naloxone methiodide administered 15 min before intraplantar capsaicin $(+R V M n l x)(n=6)$, intra-RVM muscimol administered $10 \mathrm{~min}$ before intraplantar capsaicin $(+R V M$ musc) $(n=6)$, intra-RVM muscimol administered without noxious stimulation ( $R V M$ musc alone) $(n=4)$, intra-PAG DAMGO administered as a single agent ( $P A G$ dgo alone) $(n=6)$, and intra-RVM naloxone methiodide administered $15 \mathrm{~min}$ before intra-PAG DAMGO $(+R V M$ $n l x)(n=6)$.

Relatively intense nociceptor stimulation is required to induce this form of antinociception because the response of the jawopening reflex to low-dose $(25 \mu \mathrm{g})$ capsaicin was not significantly different from the response to vehicle; at least $100 \mu \mathrm{g}$ was needed for a statistically significant antinociceptive effect. In comparison, capsaicin-induced secondary hyperalgesia, which is also dependent on activation of nociceptors, has been demonstrated in the rat with doses as low as $1 \mu \mathrm{g}$ (Gilchrist et al., 1996). The observation that local anesthetic prevented capsaicin-induced antinociception but did not reverse this effect suggests that noxious stimulation-induced antinociception is initiated by primary afferent activity but is sustained by other mechanisms.

The blockade of noxious stimulus-induced antinociception by intra-accumbens naloxone is similar to our previous findings that this same treatment also blocked heterosegmental antinociception induced by a number of different spinal interventions, including intrathecally administered DAMGO (Gear and Levine, 1995), leading us to suggest that the same ascending nociceptive control pathway mediates the antinociceptive effects of noxious stimuli. This suggestion is further supported by the observation that intra-accumbens flupentixol also blocks both noxious stimulus-induced antinociception and the antinociceptive effect of spinal intrathecal DAMGO (R. W. Gear and J. D. Levine, unpublished data).

Importantly, the applicability of these findings to the awake state was confirmed in the current study using a different nociceptive assay, the paw-withdrawal reflex. Injection of capsaicin into a forepaw induced heterosegmental antinociception, as indicated by increased thresholds for withdrawal from a mechanical stimulus applied to the two hindpaws. Furthermore, this effect
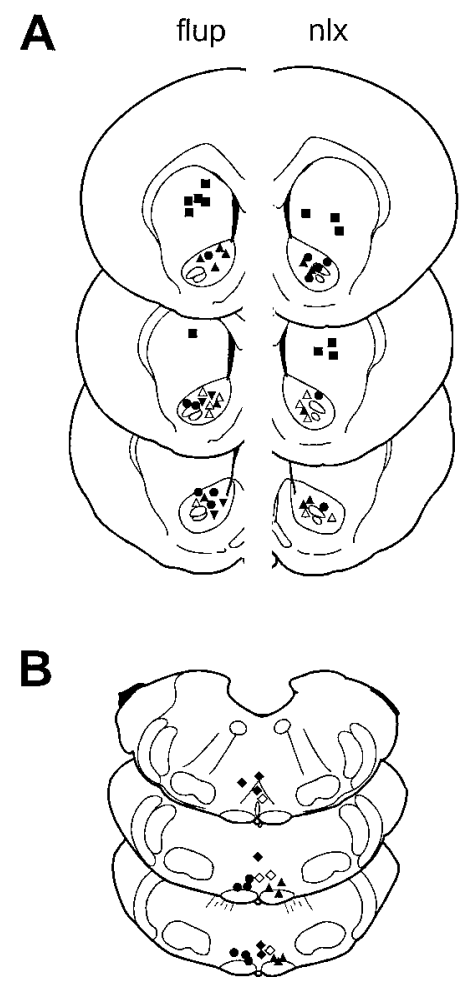

Figure 5. Supraspinal injection sites. One side is depicted for each bilateral injection on drawings adapted from the atlas of Paxinos and Watson (1986). A, Nucleus accumbens injection sites, left ( flup), flupentixol injection sites; right ( $n l x)$, naloxone methiodide injection sites. $\boldsymbol{\Delta}, \triangle$, Onsite injection before capsaicin administration in anesthetized or awake animals, respectively; $\mathbf{\nabla}$, onsite injection before hindpaw immersion in $50^{\circ} \mathrm{C}$ water bath anesthetized or awake animals, respectively (sites for naloxone methiodide in anesthetized animals not shown); $\mathbf{\square}$, off-site injection before intraplantar capsaicin administration; $\boldsymbol{\bullet}$, injection given in the absence of noxious stimulation. $B$, Rostroventral medulla injection sites. $\mathbf{\Lambda}$, Naloxone methiodide before intraplantar capsaicin administration; $\bullet$, naloxone methiodide given before intra-PAG DAMGO administration; $\diamond$, muscimol (single injections) given before intraplantar capsaicin administration; $\diamond$, without noxious stimulation.

was blocked by intra-accumbens injection of the same doses of either flupentixol or naloxone used in the experiments performed under anesthesia. Thus, the proposed ascending nociceptive control circuit appears to be active in awake as well as anesthetized animals.

Because the nociceptive assays used in the current study rely on trigeminal or spinal reflexes, by implication there must be a descending efferent circuit from the nucleus accumbens that projects either directly or via intermediate synaptic relays to these trigeminal and spinal nociceptive reflexes. Although direct projections from nucleus accumbens to the medial rostroventral medulla have not been reported, nucleus accumbens sends projections to many areas of the brain [e.g., the hypothalamus (Groenewegen and Russchen, 1984)], which, in turn, send projections to the brainstem (Sim and Joseph, 1991). Intra-RVM naloxone blocked the antinociceptive effect of intra-PAG DAMGO but failed to attenuate capsaicin-induced antinociception, suggesting that antinociceptive circuits arising in the PAG that are mediated by RVM endogenous opioids (Kiefel et al., 1993; Roychowdhury and Fields, 1996) do not mediate noxious stimulation-induced antinociception. However, intra-RVM administration of the $\mathrm{GABA}_{\mathrm{A}}$-receptor agonist muscimol blocked noxious stimulus- 
induced antinociception, suggesting that heterosegmental antinociception results from nonopioid inhibition of tonic GABAergic

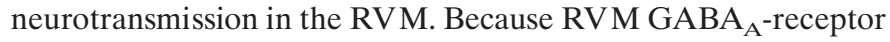
agonists induce hyperalgesia if given in sufficiently high doses (Drower and Hammond, 1988; Heinricher and Kaplan, 1991), we injected muscimol in the absence of noxious stimulation as a control. This treatment did not enhance the jaw-opening reflex significantly above baseline, suggesting that the dose used in our experiments does not produce hyperalgesia. To our knowledge, these experiments are the first demonstration that noxious stimuli are able to activate a major brainstem component of a descending pain modulation system to produce antinociception. Interestingly, this activation does not appear to involve endogenous opioids in the RVM, suggesting lack of involvement of another major component of this system, the PAG.

Although noxious stimulation-induced heterosegmental antinociception has been demonstrated previously, most notably in studies elucidating the mechanisms of the diffuse noxious inhibitory controls (DNIC) (Le Bars et al., 1979, 1992; Kraus et al., 1981; Dickenson and Le Bars, 1983), the novelty of the current findings is supported by observations that DNIC is mediated in the caudal medulla and does not require brain circuitry rostral to that point (Bouhassira et al., 1992, 1995), including, specifically, the RVM (Bouhassira et al., 1993). Thus, our findings that noxious stimulation-induced antinociception was blocked by interventions administered to nucleus accumbens as well as the RVM are inconsistent with mediation by DNIC.

The ability of intra-accumbens flupentixol to block noxious stimulus-induced antinociception suggests that painful stimuli increase mesolimbic dopamine release, although it is possible that dopamine in nucleus accumbens plays a permissive role, modulating the input of circuits such as glutamatergic corticostriate projections. This observation appears to contradict the prevailing view that dopamine in nucleus accumbens mediates responses only to appetitive or rewarding stimuli (Koob, 1996; but see Salamone et al., 1997). On the other hand, the well documented association between nucleus accumbens dopamine levels and the reinforcing properties of stimuli suggests an explanation for painseeking behavior, which, consistent with our observation that intra-accumbens naloxone also blocks noxious stimulus-induced antinociception, have been found to respond favorably to naltrexone treatment (Casner et al., 1996; Roth et al., 1996).

Capsaicin-induced attenuation of the jaw-opening reflex was unaffected in anesthetized animals with stress axis lesions (i.e., either hypophysectomized or adrenalectomized), and plasma corticosterone levels in intact animals did not change when measured before capsaicin injection and again after the onset of antinociception (data not shown). It is possible that our findings represent a previously unreported form of stress-induced analgesia (SIA) and, if so, add new information with regard to mechanisms of activation of SIA and to the supraspinal circuitry mediating SIA. Thus, although we cannot exclude stress as a factor, neither have we have been able to demonstrate that noxious stimulus-induced antinociception is dependent on stress.

In summary, we show that intense chemical or thermal noxious stimulation induces pain modulation by an ascending nociceptive control and that this effect depends on both opioid and dopamine links in the nucleus accumbens. In addition, our data suggest that noxious stimulus-induced antinociception results from a nonopioid-mediated reduction in RVM GABAergic neurotransmission. These findings add substantially to our knowledge of the mechanisms of physiologically activated pain modulation and indicate that the mesolimbic system of reward responds to aversive, as well as to positive, stimuli.

\section{REFERENCES}

Altier N, Stewart J (1993) Intra-VTA infusions of the substance P analogue, DiMe-C7, and intra-accumbens infusions of amphetamine induce analgesia in the formalin test for tonic pain. Brain Res 628:279-285.

Basbaum AI, Fields HL (1978) Endogenous pain control mechanisms: review and hypothesis. Ann Neurol 4:451-462.

Bouhassira D, Villanueva L, Bing Z, Le Bars D (1992) Involvement of the subnucleus reticularis dorsalis in diffuse noxious inhibitory controls in the rat. Brain Res 595:353-357.

Bouhassira D, Chitour D, Villanueva L, Le Bars D (1993) Morphine and diffuse noxious inhibitory controls in the rat: effects of lesions of the rostral ventromedial medulla. Eur J Pharmacol 232:207-215.

Bouhassira D, Chitour D, Villanueva L, Le Bars D (1995) The spinal transmission of nociceptive information: modulation by the caudal medulla. Neuroscience 69:931-938.

Casner JA, Weinheimer B, Gualtieri CT (1996) Naltrexone and selfinjurious behavior: a retrospective population study. J Clin Psychopharmacol 16:389-394.

Cho HJ, Basbaum AI (1991) GABAergic circuitry in the rostral ventral medulla of the rat and its relationship to descending antinociceptive controls. J Comp Neurol 303:316-328.

Daghero AM, Bradley ELJ, Kissin I (1987) Midazolam antagonizes the analgesic effect of morphine in rats. Anesth Analg 66:944-947.

Dickenson AH, Le Bars D (1983) Diffuse noxious inhibitory controls (DNIC) involve trigeminothalamic and spinothalamic neurones in the rat. Exp Brain Res 49:174-180.

Dill RE, Costa E (1977) Behavioural dissociation of the enkephalinergic systems of nucleus accumbens and nucleus caudatus. Neuropharmacology 16:323-326.

Drower EJ, Hammond DL (1988) GABAergic modulation of nociceptive threshold: effects of THIP and bicuculline microinjected in the ventral medulla of the rat. Brain Res 450:316-324.

Fields HL, Basbaum AI (1978) Brainstem control of spinal paintransmission neurons. Annu Rev Physiol 40:217-248.

Fields HL, Basbaum AI, Clanton CH, Anderson SD (1977) Nucleus raphe magnus inhibition of spinal cord dorsal horn neurons. Brain Res 126:441-453.

Fields HL, Heinricher MM, Mason P (1991) Neurotransmitters in nociceptive modulatory circuits. Annu Rev Neurosci 14:219-245.

Gear RW, Levine JD (1995) Antinociception produced by an ascending spino-supraspinal pathway. J Neurosci 15:3154-3161.

Gilchrist HD, Allard BL, Simone DA (1996) Enhanced withdrawal responses to heat and mechanical stimuli following intraplantar injection of capsaicin in rats. Pain 67:179-188.

Groenewegen HJ, Russchen FT (1984) Organization of the efferent projections of the nucleus accumbens to pallidal, hypothalamic, and mesencephalic structures: a tracing and immunohistochemical study in the cat. J Comp Neurol 223:347-367.

Heinricher MM, Kaplan HJ (1991) GABA-mediated inhibition in rostral ventromedial medulla: role in nociceptive modulation in the lightly anesthetized rat. Pain 47:105-113.

Kiefel JM, Rossi GC, Bodnar RJ (1993) Medullary $\mu$ and $\delta$ opioid receptors modulate mesencephalic morphine analgesia in rats. Brain Res 624:151-161.

Koob GF (1996) Hedonic valence, dopamine and motivation. Mol Psychiatry 1:186-189.

Kraus E, Le Bars D, Besson JM (1981) Behavioral confirmation of "diff use noxious inhibitory controls" (DNIC) and evidence for a role of endogenous opiates. Brain Res 206:495-499.

Le Bars D, Dickenson AH, Besson JM (1979) Diff use noxious inhibitory controls (DNIC). I. Effects on dorsal horn convergent neurones in the rat. Pain 6:283-304.

Le Bars D, Villanueva L, Bouhassira D, Willer JC (1992) Diffuse noxious inhibitory controls (DNIC) in animals and in man. Patol Fiziol Eksp Ter 55-65.

Mason P, Strassman A, Maciewicz R (1985) Is the jaw-opening reflex a valid model of pain? Brain Res 357:137-146. 
Paxinos G, Watson C (1986) The rat brain in stereotaxic coordinates. New York: Academic.

Roth AS, Ostroff RB, Hoffman RE (1996) Naltrexone as a treatment for repetitive self-injurious behaviour: an open-label trial. J Clin Psychiatry 57:233-237.

Roychowdhury SM, Fields HL (1996) Endogenous opioids acting at a medullary $\mu$-opioid receptor contribute to the behavioral antinociception produced by GABA antagonism in the midbrain periaqueductal gray. Neuroscience 74:863-872.

Salamone JD, Cousins MS, Snyder BJ (1997) Behavioral functions of nucleus accumbens dopamine: empirical and conceptual problems with the anhedonia hypothesis. Neurosci Biobehav Rev 21:341-359.
Sim LJ, Joseph SA (1991) Arcuate nucleus projections to brainstem regions which modulate nociception. J Chem Neuroanat 4:97-109.

Taiwo YO, Coderre TJ, Levine JD (1989) The contribution of training to sensitivity in the nociceptive paw-withdrawal test. Brain Res 487:148-151.

Tseng LF, Wang Q (1992) Forebrain sites differentially sensitive to $\beta$-endorphin and morphine for analgesia and release of Met-enkephalin in the pentobarbital-anesthetized rat. J Pharmacol Exp Ther 261:1028-1036.

Yu LC, Han JS (1989) Involvement of arcuate nucleus of hypothalamus in the descending pathway from nucleus accumbens to periaqueductal grey subserving an antinociceptive effect. Int J Neurosci 48:71-78. 